\title{
Pesquisa de infección tuberculosa latente en personal de la salud en cuatro instituciones de salud en Santiago de Chile
}

\author{
Mariluz Hernández, Carlos Casar, Patricia García, Verónica Morales, Nora Mamani, \\ Natalia Gómez-Cofré, Patricia Pizarro y M. Elvira Balcells
}

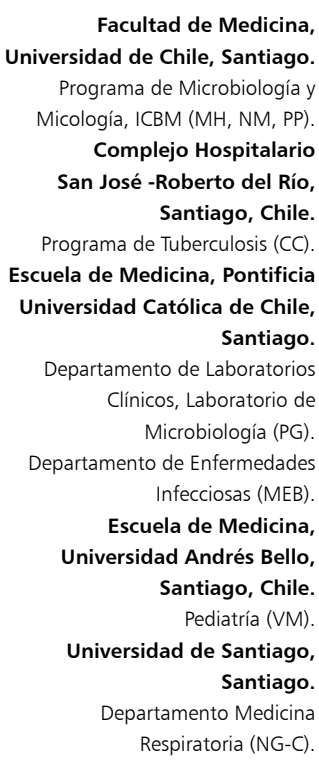

Facultad de Medicina Universidad de Chile, Santiago Programa de Microbiología y Micología, ICBM (MH, NM, PP).

Complejo Hospitalario

San José -Roberto del Río, Santiago, Chile.

Programa de Tuberculosis (CC). Escuela de Medicina, Pontificia Universidad Católica de Chile, Santiago.

Departamento de Laboratorios

Clínicos, Laboratorio de Microbiología (PG)

Departamento de Enfermedades Infecciosas (MEB).

Escuela de Medicina,

Universidad Andrés Bello, Santiago, Chile. Pediatría (VM)

Universidad de Santiago Santiago.

Departamento Medicina

Respiratoria (NG-C)

El trabajo se realizó gracias al apoyo del proyecto DI-05-09/CB de la Universidad Andrés Bello y del Laboratorio de Microbiología de la Pontificia Universidad Católica de Chile.

M.E. Balcells recibe financiamiento parcial de Proyecto FONDECYT \#

1130600, de Comisión Nacional de Investigación Científica y Tecnológica (CONICYT) Los otros autores declaran no tene conflictos de interés.

Recibido: 8 de noviembre de 2013 Aceptado: 25 de marzo de 2014

Correspondencia a: María Elvira Balcells Marty balcells@med.puc.cl

\section{Latent tuberculosis infection screening in healthcare workers in four large hospitals in Santiago, Chile}

Background: It is currently unknown which is the prevalence of latent tuberculosis infection in healthcare workers in Chile, but this group has been described as at higher risk of developing active tuberculosis than general population. Objectives: To determine the prevalence of latent tuberculosis infection in a sample of healthcare workers from at risk areas. Methodology: A cross-sectional, descriptive study, conducted in health care workers from clinical laboratories or respiratory care areas in four hospitals in Santiago. Latent tuberculosis infection detection was determined by Quantiferon ${ }^{\circledR}$ TB Gold In Tube testing (QFT). Results: QFT resulted positive in 20 of $76(26.3 \%)$ of the individuals tested. Test positivity reached $62.5 \%$ among the personnel that reported history of past TB contact in the community, $50 \%$ among the personnel who belonged to the national tuberculosis control program and 38\% among those doing induced sputum, acid fast smear or mycobacterial cultures. The proportion of individuals with positive QFT was significantly lower in those personnel who had no such risk factors $(15.7 \%$, $\mathrm{p}=0.03$ ). The proportion of latent tuberculosis infection also increased in direct relation to the age of the subject. Conclusion: Latent tuberculosis infection as detected by QFT testing was highly prevalent in healthcare workers included in the present study. Further exploring the limitations and possible scenarios for this new diagnostic tool is needed, with emphasis on health personnel at higher-risk and younger individuals.

Key words: Latent tuberculosis, interferon gamma release test, healthcare worker, tuberculosis.

Palabras claves: Infección tuberculosa latente, test de liberación de interferón gama, tuberculosis, trabajador de la salud.

\section{Introducción}

L a tuberculosis ha sido una enfermedad relevante en la historia de Chile estimándose que a inicios del siglo XX uno de cada 10 fallecimientos eran causados por esta enfermedad. Si bien la prevalencia en los últimos veinte años ha disminuido desde tasas superiores a 40 x 100.000 en 1990 a tasas de 13,2 x 100.000 en el año 2010, la letalidad se ha mantenido invariable: entre los años 2000 y 2010 han fallecido anualmente un promedio de 268 (rango 229-318) individuos por esta causa ${ }^{1}$.

Actualmente en Chile, la tuberculosis afecta principalmente a individuos con infección por VIH, vagabundos, presidiarios, contactos de tuberculosis activa, inmigrantes $\mathrm{y}$ adultos mayores ${ }^{2}$. Debido a que esta infección se transmite por aerosoles, en hospitales y centros de atención de salud, existe riesgo potencial de contagio de $M$. tuberculosis tanto hacia el personal de salud como a otros pacientes. Se ha reportado con frecuencia la transmisión de tuberculosis tras la exposición no protegida a pacientes bacilíferos y procedimientos que generan aerosoles (ej: broncoscopia, intubación oro-traqueal, esputo inducido, autopsias). Por lo tanto, cualquier individuo que trabaja en centros de atención de salud que pudiese compartir espacios aéreos con enfermos de tuberculosis se considera en riesgo de adquirir esta infección, además del personal de laboratorio que procesa muestras respiratorias y cultivos $^{3}$. Debido a que los pacientes con tuberculosis están concentrados en estos centros, el personal de la salud o que labora en estas instituciones tiene mayor riesgo que la población general de exponerse a la tuberculosis, infectarse y por ende de presentar la enfermedad.

En Chile existen muy pocos registros epidemiológicos de incidencia de tuberculosis en el personal de salud. En el año 1995, Valenzuela y cols., reportaron un riesgo de tuberculosis activa seis veces superior en el personal de la salud procedente de instituciones con riesgo evidente, al compararlo al mismo grupo etario de la población gene$\mathrm{ral}^{4}$. En otro estudio realizado en el personal de salud del Servicio de Salud Metropolitano Sur durante el período 2003-2006, la tasa estimada de prevalencia de tuberculosis activa en todas sus formas osciló entre 0 y 79 por 100.000 individuos; estos límites superiores excedían por mucho la prevalencia en población general ${ }^{5}$. En contraste, en el año 2008, el Programa Nacional de Control de la Tuberculosis estimó la incidencia de tuberculosis activa en el personal de la salud del área metropolitana por debajo de la tasa de incidencia de la población general ${ }^{2}$. Diversas variables ta- 
les como la virulencia de los aislados predominantemente circulantes en un año o área determinada, diferencias en la intensidad de la exposición, condiciones de bioseguridad variables en las diferentes instituciones y la existencia de sub-reporte en relación a la actividad laboral como factor de riesgo, podrían explicar estas diferencias en las estimaciones reportadas.

El uso del test de tuberculina para la detección de la infección tuberculosa latente es una estrategia antigua para evaluar la exposición en el personal de la salud y posterior implementación de estrategias preventivas tales como la quimioprofilaxis. Nuevas técnicas para la detección de infección tuberculosa in vitro, conocidas como ensayos de liberación de interferón gamma-IF $\gamma$ - (IGRA del inglésinterferon gamma release assays-) están disponibles para uso clínico en el mundo desde inicios del siglo XXI. Una de sus mayores ventajas radica en que la vacunación con BCG y la infección con la mayoría de las micobacterias no tuberculosas no interfieren en su interpretación, a diferencia de lo que sucede con la intradermorreacción con tuberculina.

En la actualidad se encuentran comercialmente disponibles las pruebas Quantiferon TB Gold $\AA$ In tube y T-SPOT®.TB que ya han sido aprobadas por la FDA en E.U.A. y organismos equivalentes en países europeos ${ }^{6-8}$. Estos ensayos no son equivalentes pero ambos evalúan inmunidad adquirida frente a Mycobacterium tuberculosis mediante la estimulación de linfocitos con antígenos específicos de esta micobacteria. Estos antígenos -ESAT-6, CFP-10- están codificados en la región cromosomal RD1 que está ausente en Mycobacterium bovis, en la cepa vaccinal BCG y en la mayoría de las micobacterias no tuberculosas, a excepción de $M$. kansasii, $M$. szulgai y M. marinum. El test de Quantiferon TB Gold $\AA \mathrm{y}$ Gold $₫ I n$ tube, ha sido probado en Chile desde el año 2006 , tanto en niños como en adultos. Se ha estudiado en población adulta portadora de VIH en Santiago ${ }^{9}$ y niños en contacto con tuberculosis en el área Norte y Sur de la Región Metropolitana ${ }^{10}$. Actualmente estos tests se emplean fundamentalmente como prueba diagnóstica complementaria para la pesquisa de tuberculosis latente en el contexto clínico de pacientes con enfermedades reumatológicas, otros pacientes inmunocomprometidos y pacientes oftalmológicos.

Este estudio tiene por objetivo evaluar la prevalencia de tuberculosis latente en un grupo de trabajadores de la salud con diferentes riesgos de exposición en Santiago de Chile y es el primero en emplear Quantiferon TB Gold $® I n$ Tube para este propósito en esta población.

\section{Pacientes y Métodos}

El estudio se realizó en 76 funcionarios pertenecientes a cuatro instituciones de salud de la Región Metropo- litana: Red de Salud UC, Complejo Hospitalario San José, Hospital de Niños Roberto del Río, y el Programa de Microbiología y Micología del Instituto de Ciencias Biomédicas de la Facultad de Medicina Universidad de Chile, entre mayo de 2010 y julio de 2011. Se contó con la autorización de los Comités de Ética correspondientes en todas las instituciones participantes y se cumplió con aquellos requisitos y/o consentimientos exigidos en cada centro. Junto a la toma de muestra de sangre, los participantes contestaron una breve encuesta estructurada que incluía: variables demográficas básicas, tipo de trabajo, años de vinculación laboral, antecedente de enfermedad tuberculosa, otras co-morbilidades, tuberculinas previas $\mathrm{y}$ antecedente de contacto previo en la comunidad con individuos con tuberculosis activa. Esta encuesta fue anonimizada.

La prueba de Quantiferon TB Gold $\AA$ In Tube (Cellestis, Australia) -en adelante QFT-, se realizó de acuerdo a las instrucciones del fabricante: se extrajo $1 \mathrm{ml}$ de sangre total para cada uno de tres tubos heparinizados especiales provistos por el fabricante: uno con mitógeno, otro con antígenos de tuberculosis -TB- (ESAT-6, CFP-10, TB 7,7) y el tercero un control negativo -NIL-. Los tubos se agitaron inmediatamente y se incubaron antes de $2 \mathrm{~h}$ y nueva agitación por otras $16-24 \mathrm{~h}$ a $37^{\circ} \mathrm{C}$, momento en que se recolectó y conservó el plasma a $-20^{\circ} \mathrm{C}$ hasta la determinación de la concentración de IF $\gamma$. La determinación de IF $\gamma$ se realizó mediante método de ELISA con kit provisto por Cellestis ${ }^{\circledR}$, bajo sus recomendaciones y realizada antes de tres meses del momento de la conservación del plasma. $\mathrm{Su}$ interpretación se realizó de acuerdo a instrucciones del fabricante, con el software QFT v2.62. Los valores se registraron en $\mathrm{UI} / \mathrm{mL}$. Los resultados se consideraron válidos, si la producción de IF $\gamma$ frente a mitógeno menos NIL fue $\geq 0,5 \mathrm{UI} / \mathrm{mL}$ y el NIL $<8 \mathrm{UI} / \mathrm{mL}$, o en aquellos con producción de IF $\gamma$ frente a micobacterias claramente superior al NIL, con cualquier valor de mitógeno. El resultado final informado para el valor de IF $\gamma$ se obtiene al sustraer del valor obtenido en el tubo de antígenos TB, el valor basal de IF $\gamma$ en el tubo NIL; se consideraron positivos los resultados $\geq 0,35 \mathrm{UI} / \mathrm{mL}$ y $\geq 25 \%$ NIL.

Considerando que la interpretación de esta prueba en trabajadores de la salud y la conducta a seguir con los resultados no está normada en Chile a la fecha de este estudio, y que la quimioprofilaxis no se emplea rutinariamente en el adulto, el grupo investigador entregó sus recomendaciones por escrito a cada institución, que en resumen consistían en:

- Recomendar radiografía de tórax para todos los individuos con resultados positivos y realizar estudio para descartar enfermedad activa en los sintomáticos respiratorios.

- Sugerencia de iniciar quimioprofilaxis con isoniazida en las personas con resultados positivos que presen- 
Tabla 1. Porcentaje de positividad en el test de Quantiferon TB Gold®/n Tube (QFT) en los individuos estudiados según grupo etario y antecedentes de riesgo

declarados (contacto extra-hospitalario con tuberculosis y/o prestación de servicio en el Programa Nacional de Tuberculosis)

\begin{tabular}{|lcccccc}
$\begin{array}{l}\text { Grupo etario } \\
\text { (rango de años) }\end{array}$ & $\begin{array}{c}\text { Total QFT positivos/ } \\
\text { total de individuos } \\
\text { estudiados; } \%\end{array}$ & $\begin{array}{c}\text { QFT positivos/ total } \\
\text { de individuos con } \\
\text { antecedente de } \\
\text { riesgo; } \%\end{array}$ & $\begin{array}{c}\text { Test QFT positivos/ } \\
\text { total de individuos } \\
\text { sin antecedente de } \\
\text { riesgo; \% }\end{array}$ \\
\hline $20-29$ & $0 / 22$ & $(0)$ & $0 / 4$ & $(0)$ & $0 / 18$ & $(0)$ \\
$30-39$ & $1 / 13$ & $(7,7)$ & $1 / 4$ & $(25)$ & $0 / 9$ & $(0)$ \\
$40-49$ & $3 / 17$ & $(17,6)$ & $2 / 5$ & $(40)$ & $1 / 12$ & $(8,3)$ \\
$50-59$ & $10 / 15$ & $(66,7)$ & $5 / 7$ & $(71,4)$ & $5 / 8$ & $(62,5)$ \\
$60-69$ & $5 / 6$ & $(83,3)$ & $3 / 3$ & $(100)$ & $2 / 3$ & $(66,6)$ \\
Sin edad registrada & $1 / 3$ & $(33,3)$ & $1 / 2$ & $(50)$ & $0 / 1$ & $(0)$ \\
Total & $\mathbf{2 0 / 7 6}$ & $(\mathbf{2 6 , 3 )}$ & $\mathbf{1 2} / \mathbf{2 5}$ & $\mathbf{( 4 8 )}$ & $\mathbf{8} / \mathbf{5 1}$ & $(\mathbf{1 5}, \mathbf{7})$ \\
\hline
\end{tabular}

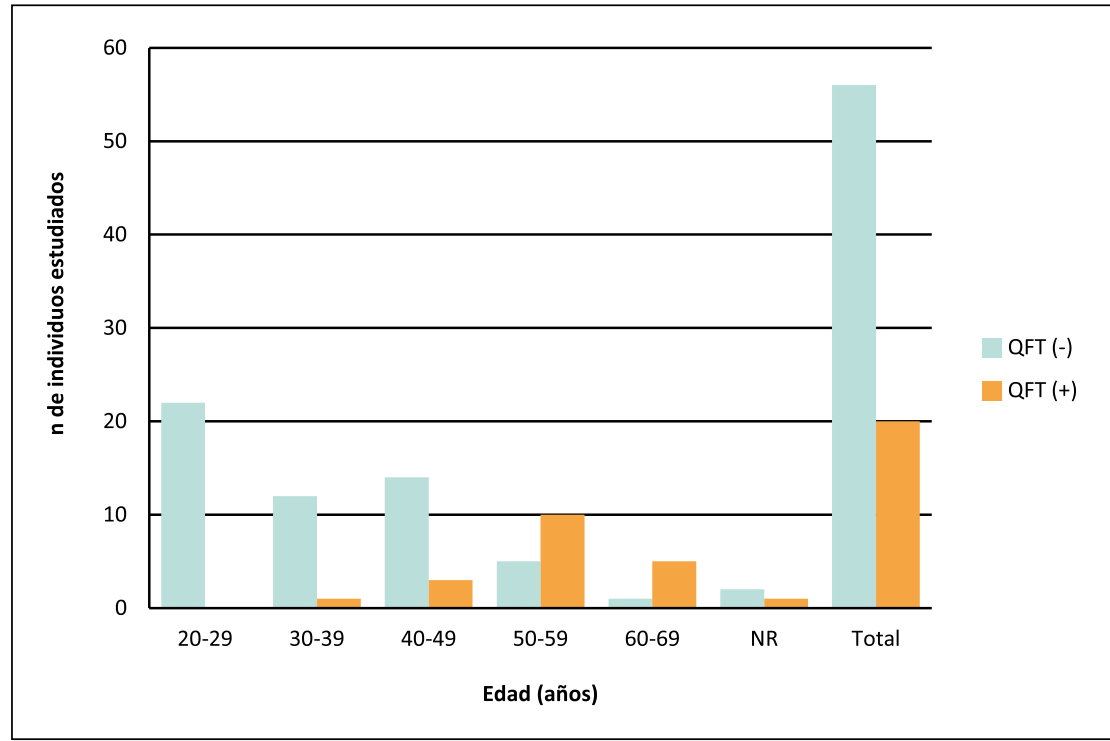

Figura 1. Frecuencia absoluta de individuos QFT (+) según grupo etario estudiado. NR: Edad no registrada.

\section{Resultados}

Participaron en total 76 funcionarios voluntarios que incluían tanto médicos, enfermeras, kinesiólogos, tecnólogos, auxiliares técnicos, como personal administrativo, con la siguiente distribución por área de trabajo: 59 (77,6\%) trabajadores de laboratorios clínicos (urgencia, microbiología y micobacterias), 14 (18,4\%) de servicios de broncopulmonar y $3(3,9 \%)$ pertenecientes a otras áreas.

El rango de edad de los participantes fue entre $20 \mathrm{y}$ 69 años, con una mediana 40 años y $64 \%$ eran mujeres. La encuesta fue respondida por 75 de los 76 funcionarios estudiados.

El resultado del QFT fue positivo en 20 del total de 76 individuos estudiados, lo que corresponde a $26,3 \%$. No se obtuvieron resultados indeterminados ni se encontraron diferencias en positividad por género: $7 / 27(25,9 \%)$ en hombres, 13/ 49 (26,5\%) en mujeres. La prevalencia de infección tuberculosa latente fue aumentando en forma significativa a mayor edad del individuo estudiado $(\mathrm{p}<0,001)$ (Tabla 1 y Figura 1$).$

Se destaca que entre los encuestados 16/75 (21,3\%) manifestaron haber tenido contacto extra-hospitalario con familiar o persona cercana con tuberculosis y de éstos, al menos 7 precisaron que fueron contactos intradomiciliarios. En ese grupo, 10/16 (62,5\%) resultaron QTF positivos. Igualmente, dos individuos del total de estudiados refirieron haber tenido tuberculosis en su infancia o juventud (1 de 2 con prueba positiva). En aquellos que no refirieron antecedente de contacto extrahospitalario con un familiar o cercano con tuberculosis, $16,9 \%$ resultaron QFT positivo (10/59). La diferencia de positividad entre aquellos que refirieron y los que no refirieron contacto extra-hospitalario fue estadísticamente significativa $(\mathrm{p}=0,003)$.

Del total de encuestados, 14/75 trabajadores de la salud declararon pertenecer o haber pertenecido al programa de tuberculosis (n: 14), de ellos 50\% (n: 7) tuvieron la prueba de QFT positiva versus 21,3\% (13/61) de los que nunca pertenecieron (p: 0,028). El tiempo de servicio en el programa de tuberculosis fue declarado por 12 personas y en promedio fue de 12,9 años (mediana 7 años, rango: 0,5-45 años).

La positividad del test de QFT en aquellos individuos que no refirieron antecedentes de contacto extra-hospitalario con enfermos de tuberculosis ni declararon haber pertenecido o pertenecer al programa de tuberculosis fue de $15,7 \%(8 / 51)$, todos los casos positivos eran mayores de 46 años y la mitad de ellos con valores de IF $\gamma$ muy elevados (mayores a $10 \mathrm{UI} / \mathrm{mL}$ ).

Según la profesión u oficio, la positividad de QFT se presentó en $8 / 30(26,7 \%)$ técnicos paramédicos o auxiliares de laboratorio, $6 / 28(21,4 \%)$ tecnólogos médicos, 2/4 
médicos, $1 / 5$ enfermeras, $0 / 2$ administrativos, $0 / 2$ y $3 / 4$ de otras profesiones u oficios no especificados.

En las actividades con riesgo evidente, se encontró que $3 / 7(42,8 \%)$ de los funcionarios que realizaban esputo inducido y $6 / 15$ (40\%) de los funcionarios que realizaban baciloscopia, cultivo de Koch y/o replicación de colonias de micobacterias resultaron positivos para QFT, dos de ellos con valores de IF $\gamma$ sobre $6 \mathrm{UI} / \mathrm{mL}$.

Cinco $(6,6 \%)$ del total de individuos estudiados y 4 (20\%) de aquellos con test positivos obtuvieron resultados en los rangos de IF $\gamma$ que algunos autores han denominado "borderline" (0,2-0,8 UI/mL), ya que pudiesen estar sujetos más fácilmente a reversiones o conversiones- en relación al corte de positividad- en las repeticiones y el seguimiento $^{12-14}$ (Tabla 2).

De los funcionarios estudiados, 35/75 (46,6\%) refirieron haberse realizado una intradermo-reacción de tuberculina en algún momento de su vida, de los cuales $8 / 35(22,8 \%)$ recuerdan haberla tenido positiva y de ellos, $3 / 8(37 \%)$ presentaron QTF positivo. En el personal que declaró haber tenido antecedentes de pertenecer o haber pertenecido al programa de tuberculosis, sólo 7/14 (50\%) refería haberse realizado alguna vez una tuberculina.

Hasta donde se pudo evaluar en el presente estudio, ninguno de los individuos estudiados recibió quimioprofilaxis antituberculosa y a la fecha de envío del manuscrito para esta publicación no se había presentado en ellos caso alguno de tuberculosis activa.

\section{Discusión}

Se ha estimado en países de bajos y medianos ingresos que la prevalencia de infección tuberculosa latente en el personal de la salud puede llegar a 54\% (33-79\%) ${ }^{11}$. El porcentaje de positividad global encontrado en el presente estudio (26,3\%) es discretamente más bajo que el límite inferior de esas cifras; sin embargo, cuando se analizan los resultados en los funcionarios que realizan actividades de mayor riesgo tales como realización de esputo inducido, baciloscopias o cultivo de micobacterias, o que pertenecen o han pertenecido al programa de tuberculosis, se observan cifras significativamente más altas, similares a lo descrito. Observamos además en este estudio, cómo el porcentaje de positividad aumenta con la edad, lo que refleja un riesgo acumulado en el tiempo o relacionado a las mayores prevalencias de tuberculosis de la población general en años y décadas previos.

Se desconoce si las infecciones latentes encontradas en estos individuos efectivamente corresponden a infecciones antiguas o son virajes recientes, debido a la ausencia de controles previos cercanos y/o anuales. Debido a que la tasa de infección tuberculosa latente en la población general en Chile no está recientemente estudiada ni
Tabla 2. Rango de valores de IFN- $\gamma$ según resultado de Quantiferon Gold In tube (QFT) en personal de la salud que resultó en rango positivo ( $\geq 0,35 \mathrm{UI} / \mathrm{ml})$

\begin{tabular}{ccccc|}
$\begin{array}{c}\text { Rango de IF } \gamma \\
\text { en } \mathrm{UI} / \mathbf{m L}\end{array}$ & \multicolumn{2}{c}{$\begin{array}{c}\mathbf{n} \text { de test (+) en ese rango/ } \\
\mathbf{n} \text { total de individuos testeados }\end{array}$} & $\begin{array}{c}\boldsymbol{n} \text { n de test (+) en ese rango/ } \\
\mathbf{n} \text { total de individuos QFT (+) }\end{array}$ \\
$\geq 0,35$ a 0,8 & $4 / 76$ & $(5,3 \%)$ & $4 / 20$ & $(20 \%)$ \\
$>0,8 \mathrm{a}<1$ & $1 / 76$ & $(1,3 \%)$ & $1 / 20$ & $(5 \%)$ \\
$\geq 1 \mathrm{a}<3$ & $7 / 76$ & $(9,2 \%)$ & $7 / 20$ & $(35 \%)$ \\
$\geq 3 \mathrm{a}<6$ & $1 / 76$ & $(1,3 \%)$ & $1 / 20$ & $(5 \%)$ \\
$\geq 6 \mathrm{a}<10$ & $2 / 76$ & $(2,6 \%)$ & $2 / 20$ & $(10 \%)$ \\
$\geq 10 \mathrm{UI} / \mathrm{ml}$ & $5 / 76$ & $(6,6 \%)$ & $5 / 20$ & $(25 \%)$ \\
\hline
\end{tabular}

definida por edad, no podemos precisar qué tanto difiere el personal de la salud incluido en el presente trabajo con la población general a edades similares, y esto es una de las limitaciones del presente estudio. Por otra parte un número importante de los evaluados $(21,3 \%)$ refirió contacto pasado con individuos con tuberculosis activa fuera del ambiente hospitalario. Esto coincide con estudios previos que han demostrado que no todos los casos de tuberculosis en el personal de la salud son atribuibles a exposición laboral y ésta explicaría entre 32 y $42 \%$ de los casos de tuberculosis en el personal de la salud ${ }^{12,13}$.

Los factores de riesgo para adquirir una infección tuberculosa en los trabajadores de la salud están relacionados con factores ambientales tales como el número de pacientes tuberculosos examinados, las características del trabajo y del lugar de trabajo (siendo mayor en terapeutas respiratorios, personal que trabaja en broncoscopias y autopsias, enfermeras, residentes y estudiantes de pregrado), el tiempo que transcurre entre el ingreso y el diagnóstico del paciente con tuberculosis, el porcentaje de pacientes con aislados multi-resistentes, el acceso a sistemas de ventilación apropiados, el tipo de barreras de protección empleadas, la falta de adherencia a las precauciones para evitar la diseminación por aerosoles y también los factores de riesgo individuales del funcionario como la presencia de desnutrición o inmunosupresión ${ }^{14}$.

Al igual que sucede en la población general, las infecciones de reciente adquisición en el personal de la salud tienen el mayor riesgo de progresar a enfermedad tuberculosa activa; por lo tanto, es racional sustentar que en el personal de la salud los esfuerzos deben centrarse en detectar infecciones recientes y, en particular, en aquellos individuos más jóvenes. Esto requeriría promover activamente la realización de un test para detección de infección tuberculosa latente al ingreso al programa laboral, para conocer el status basal, pero además mantener una vigilancia periódica que permita detectar los virajes en aquellos individuos que se mantienen en riesgo de exposición, categoría que debiera estar claramente definida. Por otra 
parte, en aquellos funcionarios de la salud en que ocurra una exposición accidental a pacientes o muestras positivas para $M$. tuberculosis, se debiera repetir el test de infección tuberculosa latente -tomando en consideración los períodos de ventana- e individualizar la quimioprofilaxis en caso de viraje de éste. La norma actual en Chile no es clara en relación al tipo de seguimiento que se debe hacer al personal de salud en riesgo de adquirir infección tuberculosa ${ }^{14,15}$.

Los estudios realizados en Chile en el personal de la salud y los índices de infección tuberculosa detectados en nuestro estudio apoyan la necesidad de continuar vigilando esta situación, idealmente en investigaciones dirigidas o respaldadas por el Programa Nacional de Tuberculosis, de modo que lleven a una normativa unificada para la salud del personal. Estos resultados, sumados a estudios de costo-efectividad, permitirían tomar decisiones fundamentadas acerca de la normativa de seguimiento en el personal de la salud y los beneficios de incorporar los IGRA por sobre la tuberculina.

Los IGRA están siendo también utilizados en países desarrollados como herramienta de vigilancia de infección por $M$. tuberculosis en el personal de la salud ${ }^{6-8}$. Sin embargo, en la actualidad se ha ido haciendo claro que también existen limitaciones y dificultades en la interpretación de estos tests que deben ser tomadas en consideración antes de su implementación en este escenario, tales como la poca claridad del efecto booster de los tests de tuberculina previos, las definiciones del nivel de corte positivo o "cut-off" y la reproducibilidad de los tests. En efecto, existe evidencia débil respecto a que el test de tuberculina cutáneo podría afectar la respuesta de los IGRA a partir del tercer día de aplicado y este efecto aparentemente podría persistir varios meses ${ }^{16}$. Por otra parte, estudios longitudinales con IGRA han demostrado que en los mismos sujetos ocurren conversiones y reversiones, y el determinar si estos cambios son inespecíficos, expresión de infecciones recientemente adquiridas, o bien reflejan erradicación de la infección, permanece aún poco claro ${ }^{17,18}$. En relación a la variabilidad intrasujeto, resultados inconsistentes han sido descritos con mayor frecuencia para QFT que para T-SPOT $® . T B$. En una cohorte de 35 trabajadores de la salud testeados en forma semanal durante cuatro semanas en Alemania, se encontró una variabilidad de $28,6 \%$ (tres conversiones, seis reversiones) para QFT versus 8,6\% (tres reversiones) para T-SPOT $® . T B(p<0,001)^{19}$. En un estudio reciente y de mayor escala realizado en la población general, en el cual se realizó repetición de los test QFT seriados, aunque la concordancia entre test repetidos fue alta $(\mathrm{k}=0,84)$ hubo discordancia en $8 \%$ de 366 sujetos y el rango de la variabilidad intra-individual al repetir los test fue de $\pm 0,24 \mathrm{UI} / \mathrm{mL}$ (coeficiente de variación de $27 \%$ ) para aquellas personas cuya respuesta QFT inicial se encontraba entre 0,25 y $0,80 \mathrm{UI} / \mathrm{mL}^{17}$. Esto introduce dificultades en la interpretación y adecuada definición de reversiones y virajes durante los seguimientos (anuales, o post exposición) en el personal de la salud. Es así como se ha sugerido el uso de zonas "borderline" para la interpretación de los IGRA seriados ${ }^{17,20,21}$, puntos de corte más altos para el personal de la salud para definir conversión que llegan a 0,8 o incluso $1,0 \mathrm{UI} / \mathrm{mL}^{22,23} \mathrm{y}$ el re-testear los resultados que son "borderline" positivos, en particular, en individuos con bajo riesgo real de exposición, antes de ofrecer quimioprofilaxis tuberculosa.

Otro aspecto relevante en la discusión de este tema es si efectivamente la sensibilidad y especificidad del IGRA es superior o no en relación al test cutáneo de tuberculina para el diagnóstico de infección tuberculosa latente. Si bien nuestro estudio tiene la limitante de no haber realizado tuberculina como test comparativo, existe bastante experiencia publicada al respecto. Un meta-análisis reciente que seleccionó 60 estudios publicados estimó la especificidad global de los IGRA (QFT y T-SPOT®.TB.TB®) en $98 \%$ para T-SPOT®.TB (95\% IC 86,8-99,9\%) y $100 \%$ para QFT (95\% IC 97.6-100\%) versus una especificidad de 55 a $95 \%$ para los test de tuberculina cutáneos, dependiendo del status BCG de las poblaciones estudiadas. En este meta-análisis el valor predictor negativo de progresión a enfermedad tuberculosa activa a dos años plazo fue de $97,8 \%$ para T-SPOT $® . T B$ y de $99,8 \%$ para QFT-G-IT ${ }^{23}$. En relación al valor predictor positivo la tasa de progresión a tuberculosis activa para aquellos sujetos con IGRA positivo que rechazaron la quimioprofilaxis tuberculosa fue de 2,3-3,3\% para tuberculina, 2,8-14,3\% para QFT-G IT y $3,3-10 \%$ para T.SPOT TB ${ }^{24-26}$.

En estudios en personal de la salud, en general hay más resultados positivos por test de tuberculina que por IGRA en los individuos vacunados con BCG, pero existen importantes diferencias en la probabilidad de concordancia para ambos tests según la procedencia y tipo de población estudiada ${ }^{27-29}$. En los sujetos aquí estudiados, $46 \%$ refirió antecedentes de haberse realizado la tuberculina pero en general con un recuerdo vago de sus resultados. En este grupo, sólo 3 de 8 que declararon haber tenido tuberculina positiva resultaron con QFT positivo, lo que podría indicar la menor especificidad de la tuberculina en el contexto de nuestra población que tiene alta cobertura de vacunación BCG. Es interesante destacar además que en los últimos años se ha postulado que la tuberculina evidenciaría mejor las infecciones antiguas y los IGRA las infecciones recientes y por ende las de mayor riesgo teórico de progresión ${ }^{30}$.

Diversos expertos y guías clínicas hacen énfasis en que la decisión de hacer una intradermo-reacción de tuberculina debe traer implícita una decisión predefinida de ofrecer quimiprofilaxis en los casos que resulten positivos, y una conducta similar está igualmente recomendada 
para los IGRA ${ }^{6,31}$. El Programa Nacional de Control de la Tuberculosis en Chile ha recomendado con éxito y ya por muchos años la quimioprofilaxis para el niño contacto de adultos con tuberculosis bacilífera, pero otros países en Latinoamérica sólo han incorporado la quimioprofilaxis recientemente y lejos están de plantearla en el adulto. Igualmente, en Chile no se recomienda la tuberculina ni la quimioprofilaxis con isoniazida en el adulto contacto por varias razones: la amplia cobertura con la vacuna BCG que dificulta la interpretación de la tuberculina, las altas tasas de tuberculosis presentadas en el siglo XX y por ende alta prevalencia de infección tuberculosa latente de larga data, la dificultad en discriminar las infecciones recientes con la tuberculina, el requerimiento de hacer doble test (dos pasos) para poder implementar esta técnica para seguimiento $\mathrm{y}$, por otra parte, el temido riesgo de toxicidad hepática por isoniazida ${ }^{14}$.

La prioridad del Programa de Control de la Tuberculosis en Chile ha sido por años y de acuerdo a lo recomendado por la Organización Mundial de la Salud, la detección de los enfermos bacilíferos, lo que explica los buenos resultados del mismo y el control de la enfermedad $^{15}$. Chile presenta en la actualidad tasas de tuberculosis bajo el umbral de eliminación, pero enfrenta inequidades y poblaciones heterogéneas en el riesgo de infección tuberculosa latente y tuberculosis. En breve el país estará enfrentándose a la necesidad de reevaluar la quimioprofilaxis en el adulto en algunas poblaciones, dentro de ellas el personal de salud, por este cambio favorable en las condiciones epidemiológicas y porque se dispone actualmente de pruebas como los IGRA que no tienen las principales desventajas de la tuberculina, si bien aún dejan interrogantes. Igualmente, la amenaza de la infección con aislados multi-resistentes, nos invita a revisar las normas de bioseguridad para la prevención de la infección tuberculosa y su implementación en los diferentes hospitales, ya que las vigentes requieren actualización ${ }^{14}$

En conclusión, en el personal de la salud testeado en el presente estudio, se detectó una alta prevalencia de infección tuberculosa latente por test de QFT, llegando a más de $40 \%$ en aquellos con antecedente de contacto extra-hospitalario con tuberculosis, actividades profesionales de riesgo o servicio en el Programa Nacional de Control de la Tuberculosis versus $15,7 \%$ de positividad en el personal de salud sin ninguno de esos antecedentes. Las ventajas que ofrecen los IGRA y los resultados obtenidos en este estudio nos hacen proponer que estas pruebas deben evaluarse en forma más amplia y ordenada en Chile para definir su rol tanto en la vigilancia de la salud del personal como en el Programa Nacional de Control de la Tuberculosis. No podemos comparar estos resultados con la población general ya que estas cifras se desconocen actualmente en Chile. Es necesario realizar estudios de costo efectividad, por su mayor costo comparadas a la tuberculina. Del mismo modo, es momento de poner sobre la mesa la pertinencia de la quimioprofilaxis de los diferentes adultos en riesgo, dentro de los cuales destacamos el adulto contacto cercano o intra-domiciliario de un paciente bacilífero y el personal de la salud recientemente infectado.

Agradecimientos: A CellestisInc $\AA$, ya que una parte de los test de QuantiFERON TBGold ${ }^{\circledR}$ In tuve fueron proporcionados por ellos.

\section{Resumen}

Introducción: Se desconoce en la actualidad cuál es la real prevalencia de infección tuberculosa latente en el personal de salud en Chile; sin embargo, este grupo ha sido descrito como con mayor riesgo de desarrollar tuberculosis activa que la población general. Objetivo: Determinar la prevalencia de infección tuberculosa latente en funcionarios de la salud en diferentes áreas laborales de riesgo. Metodología: Estudio de corte transversal, descriptivo, realizado en funcionarios pertenecientes a laboratorios clínicos o áreas de atención broncopulmonar de cuatro hospitales de la Región Metropolitana en quienes se hizo test de Quantiferon TB Gold®In tube(QFT). Resultados: Se evidenció infección tuberculosa latente en 20 de las 76 (26,3\%) personas estudiadas. En aquellos funcionarios que referían antecedente de contacto en el pasado en la comunidad con enfermos de tuberculosis, la positividad del test llegó a $62,5 \%$; en aquellos que pertenecían al Programa Nacional de Control de la Tuberculosis, a 50\% y en los que realizaban toma de esputo inducido, baciloscopias o cultivo de micobacterias, a 38\%. La proporción de individuos con QFT positivo fue significativamente menor en aquellos funcionarios que no tenían estos antecedentes $(15,7 \%, \mathrm{p}=0,03)$. Se encontró además una mayor proporción de infección tuberculosa latente a mayor edad del individuo estudiado. Conclusión: La infección tuberculosa latente medida por QFT resultó altamente prevalente en el personal de la salud incluido en el presente estudio. Es necesario seguir profundizando en los posibles escenarios de implementación y limitaciones del uso de esta nueva herramienta diagnóstica, haciendo énfasis en el personal de la salud de mayor riesgo y menor edad. 


\section{Referencias bibliográficas}

1.- Herrera T. Tuberculosis: Perfil Epidemiológico Actual en Chile. Memorias XXIX Congreso Chileno Nacional de Infectología Pucón 2012. http://www.sochinf.cl/sitio/templates/ sochinf2008/documentos/presentaciones congre_2012/lonquijuev/17_00_17_30.pdf (accedido 12 de septiembre de 2013).

2.- García C. Tuberculosis en grupos de riesgo en la Región Metropolitana, 2008. Rev Chil Enf Respir 2010; 26: 105-11.

3.- Jensen P A, Lambert L A, Iademarco M F, Ridzon R, CDC. Guidelines for preventing the transmission of Mycobacterium tuberculosis in health-care settings, 2005. MMWR Recomm Rep 2005; 54 (RR-17): 1-141.

4.- Valenzuela M T, Lagos A, Schroeder F, O’Ryan L. Transmisión de la tuberculosis al personal de salud. Rev Chilena Infectol 1998; 15: $280-4$.

5.- Fica A, Ramonda P; Jemenao I, Zambrano A, Cifuentes M, Febré N, et al. Tuberculosis en el personal de salud del Servicio de Salud Metropolitano Sur de Santiago, Chile. Rev Chilena Infectol 2009; 26: 34-8.

6.- Mazurek G H, Jereb J, Vernon A, LoBue P, Goldberg S, Castro K, IGRA Expert Committee, Centers for Disease Control and Prevention. Updated guidelines for using Interferon Gamma Release Assays to detect Mycobacterium tuberculosis infection-United States, 2010. MMWR Recomm Rep 2010; 59 (RR-5): 1-26.

7.- National Institute for Health and Clinical Excellence. Tuberculosis: clinical diagnosis and management of tuberculosis, and measures for its prevention and control. Nice Clinical Guidelines 117. London, 2011. Disponible en:http://www.nice.org.uk/nicemedia/ live/13422/53642/53642.pdf (accedido el 22 de noviembre de 2013).

8.- European Centre for Disease Prevention and Control. Use of interferon-gamma release assays in support of TB diagnosis. Stockholm: ECDC; 2011. Disponible en: http://ecdc.europa.eu/en/ publications/Publications/1103_GUI_IGRA.pdf (accedido el 22 de noviembre de 2013).

9.- Balcells M E, Pérez C M, Chanqueo L, Lasso M, Villanueva M, Espinoza M, et al. A comparative study of two different methods for the detection of latent tuberculosis in HIVpositive individuals in Chile. Int $\mathrm{J}$ Infect Dis 2008; 12: 645-52.

10.- Casar C, Hernández M, Castro-Rodríguez J A, Mamani N, Pizarro P, Rueda B, et al. Comparación de la producción de interferon gamma frente a diferentes antígenos de mycobacterium, entre niños contactos de tuberculosis y niños vacunados con BCG. Rev Chil Enf Respir 2008; 4: 352.

11.- Joshi R, Reingold A L,Menzies D, Pai M Tuberculosis among health-care workers in low and middle-income countries: A systematic review. PLoS Med 2006;3: e494.

12.- de Vries G, Sebek M M, Lambregts-van Weezenbeek C S. Healthcare workers with tuberculosis infected during work. Eur Respir J 2006; 28: 1216-21.

13.- Ong A, Rudoy I, González L C, Creasman J, Kawamura L M, Daley C L. Tuberculosis in healthcare workers: A molecular epidemiologic study in San Francisco. Infect Control Hosp Epidemiol 2006; 27: 453-8.

14.- Fica A, Cifuentes M, Ajenjo M C, Jemenao I, Zambrano A, Febré N, et al. Comité Consultivo de Infecciones Intrahospitalarias Sociedad Chilena de Infectología. Tuberculosis en el personal de la salud. Rev Chilena Infectol 2008; 25: 243-55.

15.- Zuñiga M, Valenzuela P, Farga V, Yáñez A, Rojas M, et al. Manual de Organización y Normas Técnicas. Programa Nacional de Control de la Tuberculosis. Ministerio de Salud. Subsecretaría de Salud Pública. 2005. Disponible en: http://web.minsal.cl/portal/url/it em/803048171acc60f8e04001011 f0148e2.pdf (accedido el 22 de noviembre de 2013).

16.- van Zyl-Smit R N, Zwerling A, Dheda K, Pai M. Within-subject variability of interferon- $\gamma$ assay results for tuberculosis and boosting effect of tuberculin skin testing: a systematic review. PLOS ONE 2009; 4: e8517.

17.- Metcalfe J Z, Cattamanchi A, McCulloch C E, Lew J, Ha N P, Graviss E A. Test variability of the Quantiferon-TBGold in-tube assay in clinical practice. Am J Respir Crit Care Med. 2013; 187: 206-11.

18.- Zanetti C, Peracchi M, Zorzi D, Fiorio S, Fallico L, Palù G. Outbreak of transient conversions of the Quantiferon-TB Gold In-Tube test in laboratory health care worker screenings. Clin Vaccine Immunol 2012; 19 : 954-60.

19.- Ringshausen F C, Nienhaus A, Torres Costa J, Knoop H, Schlösser S, Schultze-Werninghaus $\mathrm{G}$, et al. Within-subject variability of Mycobacterium tuberculosis-specific gamma interferon responses in German health care workers. Clin Vaccine Immunol 2011; 18: 1176-82.

20.- Pai M, Joshi R, Dogra S, Mendiratta D K, Narang P, Kalantri S, et al. Serial testing of health care workers for tuberculosis using interferon-gamma assay. Am J Respir Crit Care Med 2006; 174: 349-55.

21.- Schablon A, Harling M, Diel R, Ringshausen F C, Torres Costa J, Nienhaus A. Serial testing with an interferon- $\gamma$ assay in German healthcare workers. GMS Krankenhhyg Interdiszip 2010 ; 5: Doc05.

22.- Slater M L, Welland G, Pai M, Parsonnet J, Banaei N. Challenges with Quantiferon-TB Gold assay for large-scale, routine screening of US healthcare workers. Am J Respir Crit Care Med 2013; 188: 1005-10.

23.- Daley C L, Reves R R, Beard M A, Boyle J, Clark R B, Beebe J L, et al. A summary of meeting proceedings on addressing variability around the cut point in serial interferon- $\gamma$ release assay testing. Infect Control Hosp Epidemiol 2013; 34: 625-30.

24.- Diel R, Goletti D, Ferrara G, Bothamley G, Cirillo D, Kampmann B, et al. Interferon- $\gamma$ release assays for the diagnosis of latent Mycobacterium tuberculosis infection: a systematic review and meta-analysis. Eur Respir J 2011; 37: 88-99.

25.- Diel R, Loddenkemper R, Meywald-Walter K, Niemann S, Nienhaus A. Predictive value of a whole blood IFN- $\gamma$ assay for the development of active tuberculosis disease after recent infection with Mycobacterium tuberculosis. Am J Respir Crit Care Med 2008; 177: 1164-70

26.- Kik SV, Franken W P, Mensen M, Cobelens F G, Kamphorst M, Arend S M, et al. Predictive value for progression to tuberculosis by IGRA and TST in immigrant contacts. Eur Respir J 2010; 35: 1346-53.

27.- Harada N, Nakajima Y, Higuchi K, Sekiya Y, Rothel J, Mori T. Screening for tuberculosis infection using whole-blood interferon- $\gamma$ and Mantoux testing among Japanese healthcare workers. Infect Control Hosp Epidemiol 2006; 27: 442-8

28.- Pai M, Gokhale K, Joshi R, Dogra S, Kalantri S, Mendiratta D K, et al. Mycobacterium tuberculosis infection in health care workers in rural India: comparison of a whole-blood interferon $\gamma$ assay with tuberculin skin testing. JAMA 2005; 293: 2746-55.

29.- Pollock N R, Campos-Neto A, Kashino S, Napolitano D, Behar S M, Shin D, et al. Discordant Quantiferon-TB gold test results among US healthcare workers with increased risk of latent tuberculosis infection: a problem or solution? Infect Control Hosp Epidemiol 2008; 29: 878-86.

30.- Pai M, O’Brien R. Serial testing for tuberculosis: Can we make sense of $\mathrm{T}$ cell assay conversions and reversions? PLoS Med 2007; 4: e208.

31.- Horsburgh C R Jr, Rubin E J. Clinical Practice. Latent tuberculosis infection in the United States. N Engl J Med 2011; 364: 1441-8. 